\title{
A meta-analysis of the clinical efficacy and safety of Bailing capsules in the treatment of nephrotic syndrome
}

\author{
Hui Xu, Xiaozhao Li, Xiangning Yuan, Qiongjing Yuan, Wenhang Chen, Zhangzhe Peng, \\ Xiangcheng Xiao, Qiaoling Zhou \\ Department of Nephrology, Xiangya Hospital, Central South University, Changsha, China \\ Contributions: (I) Conception and design: H Xu, X Xiao, Q Zhou; (II) Administrative support: X Li, X Yuan; (III) Provision of study materials or \\ patients: H Xu, Q Yuan, W Chen, Z Peng, X Xiao; (IV) Collection and assembly of data: All authors; (V) Data analysis and interpretation: X Li, \\ W Chen, Z Peng, Q Zhou; (VI) Manuscript writing: All authors; (VII) Final approval of manuscript: All authors. \\ Correspondence to: Qiaoling Zhou; Xiangcheng Xiao. Department of Nephrology, Xiangya Hospital, Central South University, Changsha, China. \\ Email: qiaolingzhou1234@163.com; xiaoxch2000@163.com.
}

Background: Nephrotic syndrome (NS) is extremely harmful to human health. Bailing capsules can reduce proteinuria and improve renal function in patients with NS. This meta-analysis aimed to evaluate the clinical efficacy and safety of Bailing capsules in the treatment of NS.

Methods: Systematic searches of the English-language databases PubMed, Cochrane, Embase, Medline, and Web of Science, as well as the Chinese-language databases China Academic Journals Full-text Database (CJFD), Wanfang Data, and Weipu (VIP), were performed. Randomized controlled trials (RCTs) of Bailing capsules in the treatment of NS were included in the meta-analysis. The total effective rate and adverse reaction rate were evaluated with relative risk $(\mathrm{RR})$ and the quantitative data was evaluated with standardized mean difference (SMD) and 95\% confidence interval (CI). The quality of the included articles was evaluated using RevMan5.3 software, and the "meta" package of R3.5.1 software was used for all other statistical analysis.

Results: A total of 20 papers involving 819 and 824 patients in the treatment group and the control group, respectively, were included. The total effective rate and adverse reaction rate after treatment were reported in 17 and 2 articles, respectively. The total effective rate of the treatment group was 1.22 times that of the control group $\left(\mathrm{I}^{2}=0 \%\right.$, fixed-effects model, $\left.95 \% \mathrm{CI}: 1.16,1.27\right)$, and the adverse reaction rate of the treatment group was 0.22 times that of the control group $\left(\mathrm{I}^{2}=0 \%\right.$, fixed-effects model, $95 \%$ CI: 0.08 , 0.63). The levels of blood urea nitrogen (BUN) and serum creatinine (SCr) before and after treatment were reported in 15 articles, with SMDs of -0.98 ( $\mathrm{I}^{2}=50 \%$, fixed-effects model, 95\% CI: $\left.-1.10,-0.87\right)$ and -1.47 ( $\mathrm{I}^{2}=96 \%$, random-effects model, 95\% CI: -2.08, -0.86), respectively. Meanwhile, 13 articles reported the level of 24-hour proteinuria before and after treatment, with an SMD of -1.25 ( $\mathrm{I}^{2}=92 \%$, fixed effects model, 95\% CI: $-1.73,-0.78)$.

Conclusions: For NS patients, treatment with Bailing capsules can achieve higher clinical efficacy and a lower adverse reaction rate than conventional treatment in improving the function of kidney.

Keywords: Nephrotic syndrome (NS); Bailing capsule; meta-analysis

Submitted May 12, 2020. Accepted for publication Aug 18, 2020.

doi: 10.21037/apm-20-1252

View this article at: http://dx.doi.org/10.21037/apm-20-1252 


\section{Introduction}

Nephrotic syndrome (NS) is a clinical symptom of increased glomerular basement membrane permeability. NS is characterized by large amounts of proteinuria, hypoproteinemia, edema, and/or hyperlipidemia. As one of the 10 leading causes of mortality in the United States, NS poses a significant threat to human health (1). In China, a retrospective survey of 5,398 adults showed that the proportion of renal patients with clinical manifestations of NS gradually increased from $37.7 \%$ to $42.2 \%$ in the decade between 1997 and 2007 (2).

The pathogenesis of NS is relatively complicated, mainly because of the abnormal immune response and the kidney damage caused by the inflammatory response. Proteinuria, which can cause damage to glomerular intrinsic cells such as mesangial cells, podocytes, and tubulointerstitial cells is the most basic pathophysiological cause of NS. Persistent proteinuria can promote kidney damage and is closely related to the progression of chronic renal failure. Therefore, reducing proteinuria constitutes an important target for the treatment of NS. Clinically, according to the different pathologic types of nephrotic syndrome, the treatment methods of NS are different. treating the primary disease actively can delay the occurrence of secondary nephropathy, and effectively protect the function of kidney. The routine treatment and symptomatic treatment, like supplement of high-quality protein in bed for the treatment of severe edema, hypoproteinemia. Besides, the clinical treatment of NS is mainly based on suppressing the immune inflammation. The use of renin-angiotensin blockers and/ or immunosuppressants has been recommended; through inhibiting aldosterone and antidiuretic hormone secretion, altering the permeability of the glomerular basement membrane, and exerting other comprehensive effects, these drugs support diuresis and the elimination of protein in urine (3). However, the long-term use of hormone-based therapies can easily lead to complications such as secondary infection, thromboembolism, or aseptic necrosis of the femoral head, which reduce the quality of life of the patient (4).

In recent years, Chinese medicines and Chinese patent medicines have gradually drawn attention as adjuvant treatments for NS patients (3). It has been reported that traditional Chinese medicine, acupuncture, acupoint injection combined with prednisone in the treatment of NS has a good efficacy. Cordyceps sinensis is a single herb with wide clinical application in the department of nephrology.
It exhibits a variety of functions to protect the kidney, including reducing urine protein, alleviating kidney injury, regulating immune function, and promoting the recovery of renal tubular function (5). The Bailing capsules made from the hypha of cordyceps sinensis strain shows the same effect in the treatment of NS. Bailing capsules, which were independently developed in China, are a preparation made from cordyceps. A previous meta-analysis of five articles reported that Bailing capsules could reduce proteinuria and improve renal function in NS patients (6). Subsequently, a large number of clinical studies on Bailing capsules for NS were reported (7-10). However, owing to the small scale of most studies in Chinese medicine, as well as the low quality of the literature, the various empirical information contained in the clinical data has yet to be fully dissected. The clinical efficacy and safety analysis of Bailing capsules in the treatment of NS urgently need to be explored. Therefore, we conducted a meta-analysis of RCTs on the clinical efficacy and safety of Bailing capsules combined with conventional Western medicine in the treatment of NS, with the aim of producing an objective and comprehensive systematic review to guide future clinical application. We present the following article in accordance with the PRISMA reporting checklist (available at http:// dx.doi.org/10.21037/apm-20-1252).

\section{Methods}

\section{Literature search}

Systematic searches were performed of the Englishlanguage databases PubMed, Cochrane, Embase, Medline, and Web of Science, as well as the Chinese-language databases China Academic Journals Full-text Database (CJFD), Wanfang Data, and Weipu (VIP). The searches were conducted for articles published up to March 31, 2020, using a combination of subject words and keywords. English search terms included: "Corbrin or Bailing", "Nephrotic syndrome", and "NS", with the search language being limited to English; Chinese search terms included: "Kidney syndrome", "Bai Ling capsule", and "Bai Ling". The keywords in the above search terms were connected with "or", and the drugs and diseases were connected with "and". To prevent any relevant articles from being omitted from the meta-analysis, the references of the articles from the databases were retrieved through Google Scholar and other softwares. 


\section{Inclusion and exclusion criteria}

The retrieved articles were screened according to the following inclusion criteria: (I) patients with a conclusive diagnosis of NS; (II) the control group received conventional hormone therapy; (III) the experimental group was treated with Bailing capsules and other drugs based on the control group treatment; (IV) randomized clinical controlled trial; (V) the course of treatment was not limited; and (VI) the outcome indicators included any of the following: total treatment effectiveness, incidence of adverse reactions, and renal function indicators, including blood urea nitrogen (BUN) before and after treatment, serum creatinine (Scr), and 24-hour urine protein quantification before and after treatment.

The exclusion criteria were as follows: (I) young pediatric patients with NS; (II) elderly patients, or NS combined with other serious diseases; (III) animal experiments, reviews, case reports, and none clinical studies; (IV) non-RCT; or (V) the above-mentioned outcome indexes were not included.

\section{Literature screening and data extraction}

The retrieved articles were screened independently according to the inclusion and exclusion criteria by two data extractors. The titles, abstracts and full texts of the articles were read, and uniform data extraction tables were used for data extraction and to identify articles meeting the inclusion and exclusion criteria. The basic information extracted from the articles included: author, publication year, basic patient characteristics (including age, gender, and course of disease), the treatment methods, treatment time, sample size, effectiveness, adverse reaction indicators of the control and experimental groups, the mean and standard deviation of BUN, Scr, and 24-hour urine protein quantification of the two groups before and after treatment. Then, the mean and standard deviation before and after treatment were calculated according to the RevMan 5.1 manual (The Cochrane Collaboration, Copenhagen, Denmark).

\section{Literature quality evaluation}

Two researchers evaluated the quality of the literature using Cochrane's risk of bias assessment tool, which assesses the bias risk in six domains (11): (I) selection bias: including whether the random allocation method was described in depth and whether the hidden random assignment method was described; (II) implementation bias: whether the researcher and the subject were blinded, and whether sufficient information by which to judge the blinding method was included; (III) measurement bias: whether the researchers used a blinding method to evaluate the results; (IV) follow-up bias: whether the reasons for loss of followup or withdrawal were reported to ensure the completeness of the data; $(\mathrm{V})$ reporting bias: whether selective reporting of research results could be judged; (VI) whether other sources of bias could be assessed. Each bias indicator was evaluated as "low bias", "unclear", or "high bias". For inconsistencies in the literature screening, data extraction, and article quality evaluation processes, the opinion of a third researcher would be sought.

\section{Statistical analysis}

The quality of the articles was evaluated using Revman 5.3 software. The total effective and the adverse reaction rates were merged using ratio risk (RR) and 95\% confidence interval (CI). The renal function indexes of BUN, Scr, and 24-hour urine protein were merged using standardized mean difference (SMD), and $95 \%$ CI. $\mathrm{I}^{2}$ was used to evaluate heterogeneity. When $\mathrm{I}^{2} \leq 50 \%$, the fixed-effects model was used to estimate the effect value, while for $\mathrm{I}^{2}>50 \%$, the random-effects model was used. For studies with heterogeneity, sensitivity analysis was performed after eliminating the articles one by one, and a stratification analysis of different treatment times was conducted to explore the sources of heterogeneity. A funnel chart was used to evaluate the qualitative quality of the articles, and Begg's rank correlation and Egger's regression tests were used for quantitative evaluation of publication bias. The results of the effect value, forest map, publication bias, sensitivity, and other analyses were all statistically analyzed using the "meta" package of R3.5.1 software.

\section{Results}

\section{Basic information of the included articles}

Initially, 129 Chinese and English articles were retrieved. According to the inclusion and exclusion criteria, 20 Chinese articles (7-10,12-27) were finally included. No English-language articles met the criteria for inclusion. Figure 1 shows the literature search process. The 20 included articles involved 1,643 patients with NS, including 819 cases in the test group and 824 cases in the control group. The research subjects were definitely diagnosed as 


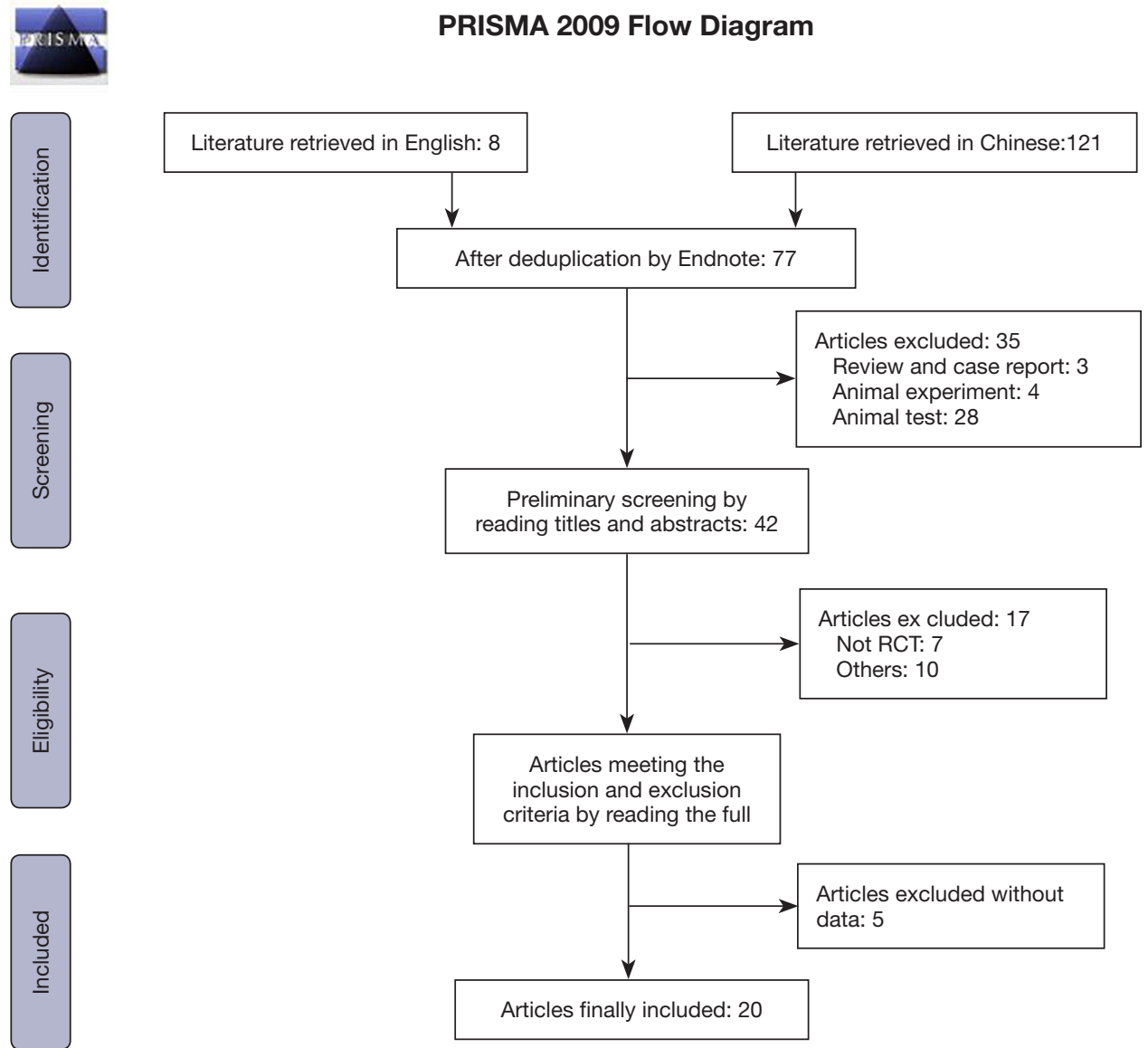

Figure 1 Flow chart of article selection.

primary NS in 6 articles $(12,21,23,25-27)$, while the subjects in the other articles were only reported as NS. Other information included in the literature is shown in Table 1.

\section{Literature quality evaluation}

All of the included articles reported that random allocation was used to avoid patient selection bias, but the levels of implementation bias, follow-up bias, reporting bias, and other biases were unclear. The results of Cochrane risk bias assessment are shown in Figure 2.

\section{Meta-analysis of the total effective rate}

The total effective rate after treatment was reported in 17 articles. There was low heterogeneity $\left(\mathrm{I}^{2}=0 \%\right)$; therefore, the fixed-effects model was used to estimate RR. The total effective rate of conventional treatment combined with the Bailing capsules method was 1.22 times (95\% CI: 1.16, 1.27) that of the control group (Figure 3).

\section{Meta-analysis of the adverse reaction rate}

Two articles reported the adverse reaction rate after treatment. There was low heterogeneity $\left(\mathrm{I}^{2}=0 \%\right)$; therefore, RR was estimated using a fixed-effects model. The adverse reaction rate in the experimental group was 0.22 times that in the control group (95\% CI: 0.08, 0.63) (Figure 4).

\section{Meta-analysis of BUN level}

The level of BUN before and after treatment was reported in 15 articles. There was low heterogeneity between the studies $\left(\mathrm{I}^{2}=50 \%\right)$; therefore, a fixed-effects model was used to estimate SMD. The difference in BUN level before and after treatment in the experimental group was significantly lower than that in the control group, with an SMD of -0.98 (95\% CI: $-1.10,-0.87$, Figure 5). 


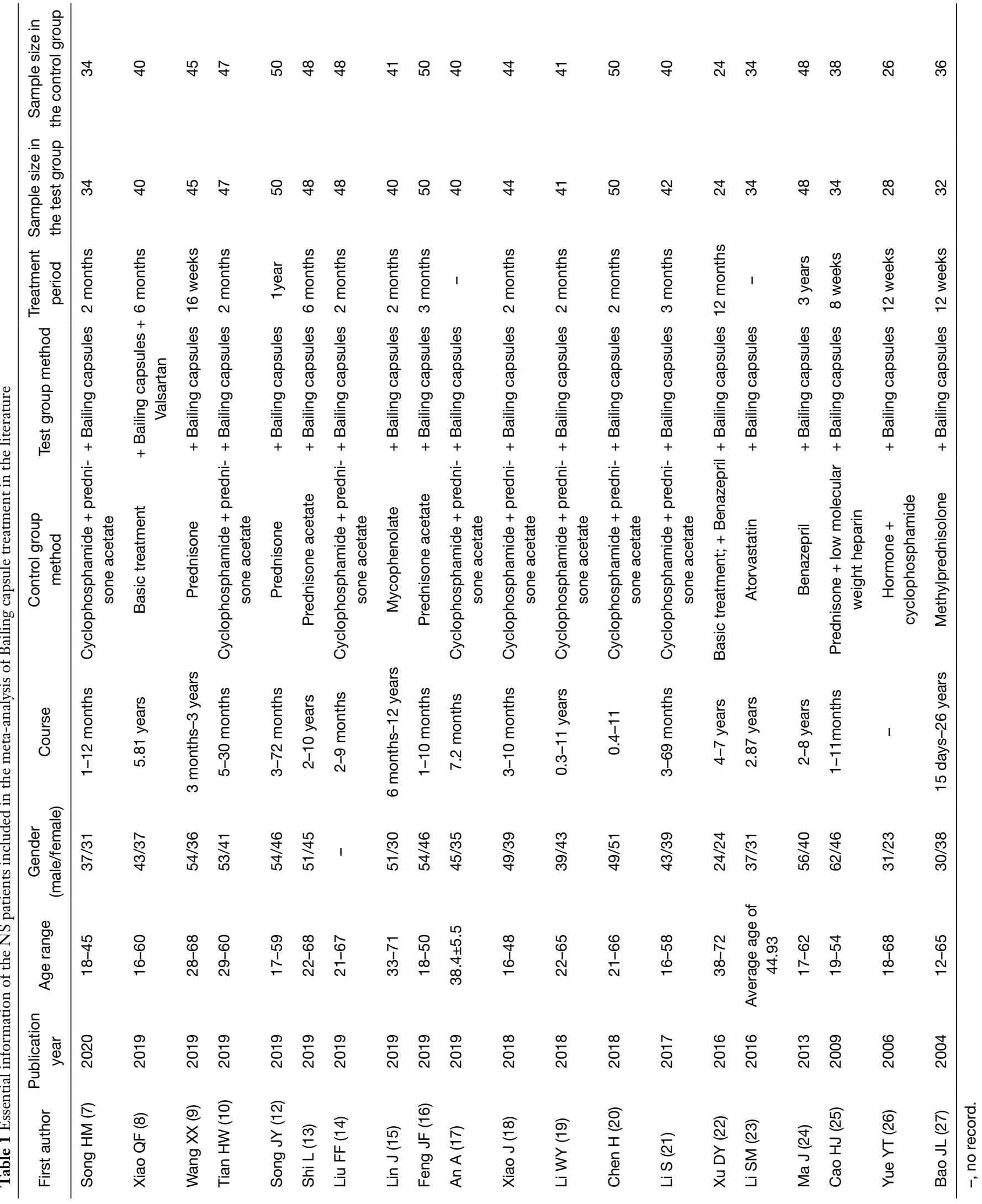




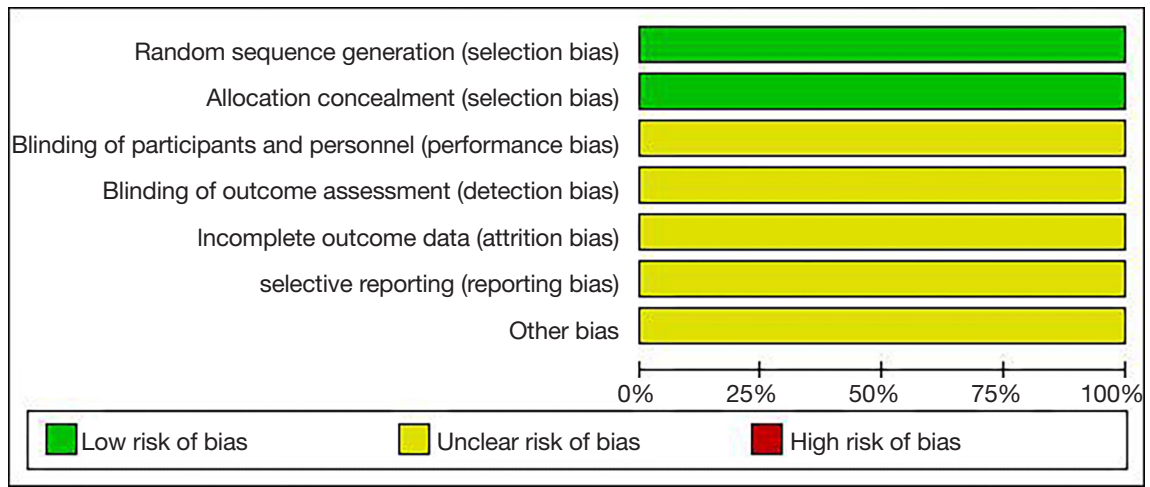

Figure 2 Quality bias evaluation of the included studies.

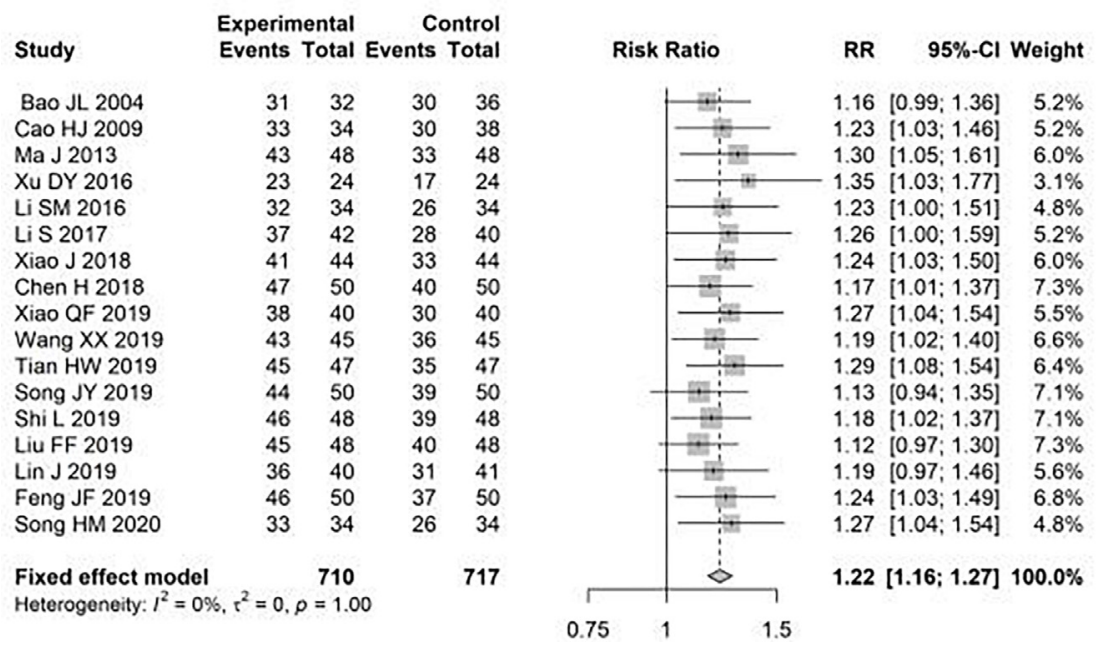

Figure 3 Forest plot of meta-analysis of the total effective rate of Bailing capsules in NS treatment. NS, nephrotic syndrome.

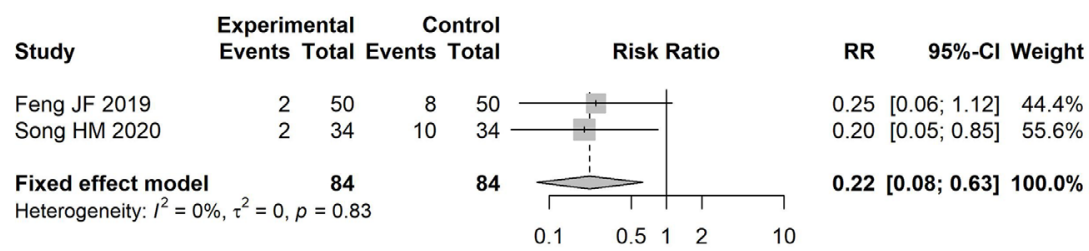

Figure 4 Forest plot of meta-analysis of the incidence of adverse reactions of Bailing capsules in NS treatment. NS, nephrotic syndrome.

\section{Meta-analysis of SCr level}

The level of SCr before and after treatment was reported in 15 articles. High heterogeneity existed between the studies $\left(\mathrm{I}^{2}=96 \%\right)$; therefore, a random-effects model was used to estimate the SMD. The difference in SCr level before and after treatment in the experimental group was significantly lower than that in the control group, with an SMD of -1.47

(95\% CI: -2.08, -0.86, Figure 6).

\section{Meta-analysis of 24-hour urine protein level}

The 24-hour urinary protein levels before and after 


\begin{tabular}{|c|c|c|c|c|c|c|c|c|c|c|}
\hline \multirow[b]{2}{*}{ Study } & \multirow[b]{2}{*}{ Total } & \multicolumn{2}{|c|}{ Experimental } & \multicolumn{3}{|r|}{ Control } & Standardised Mean & \multirow[b]{2}{*}{ SMD } & \multirow[b]{2}{*}{$95 \%-\mathrm{Cl}$} & \multirow[b]{2}{*}{ Weight } \\
\hline & & Mean & SD & Total & Mean & SD & Difference & & & \\
\hline Yue YT 2006 & 28 & -0.39 & 0.5991 & 26 & -0.09 & 0.7296 & & -0.44 & {$[-0.99 ; 0.10]$} & $4.6 \%$ \\
\hline Cao HJ 2009 & 34 & -0.11 & 2.5668 & 38 & 0.04 & 2.3931 & & -0.06 & {$[-0.52 ; 0.40]$} & $6.3 \%$ \\
\hline Li S 2017 & 42 & -3.81 & 1.2322 & 40 & -2.66 & 1.3241 & 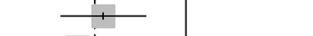 & -0.89 & {$[-1.35 ;-0.44]$} & $6.5 \%$ \\
\hline Xiao J 2018 & 44 & -2.58 & 0.6332 & 44 & -1.52 & 1.1058 & $\begin{array}{c:c}1 \\
1\end{array}$ & -1.17 & {$[-1.62 ;-0.71]$} & $6.6 \%$ \\
\hline Li WY 2018 & 41 & -2.73 & 1.2483 & 41 & -1.28 & 1.2665 & \begin{tabular}{c}
1 \\
\hdashline
\end{tabular} & -1.14 & {$[-1.61 ;-0.67]$} & $6.2 \%$ \\
\hline Chen H 2018 & 50 & -2.58 & 1.0867 & 50 & -1.52 & 1.1058 & 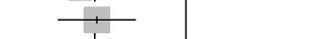 & -0.96 & {$[-1.37 ;-0.54]$} & $7.9 \%$ \\
\hline Xiao QF 2019 & 40 & -3.20 & 0.9762 & 40 & -1.90 & 1.2465 & \begin{tabular}{ll}
1 \\
\hdashline
\end{tabular} & -1.15 & {$[-1.62 ;-0.68]$} & $6.0 \%$ \\
\hline Wang XX 2019 & 45 & -12.05 & 3.1022 & 45 & -8.73 & 3.1625 & 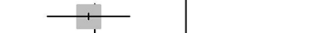 & -1.05 & {$[-1.49 ;-0.61]$} & $6.9 \%$ \\
\hline Tian HW 2019 & 47 & -3.24 & 1.0137 & 47 & -1.66 & 1.0376 & 1 & -1.53 & {$[-1.99 ;-1.07]$} & $6.3 \%$ \\
\hline Song JY 2019 & 50 & -3.95 & 1.2120 & 50 & -2.60 & 1.3727 & $T$ & -1.03 & {$[-1.45 ;-0.62]$} & $7.7 \%$ \\
\hline Shi L 2019 & 48 & -2.66 & 1.1418 & 48 & -1.16 & 1.9421 & + & -0.93 & {$[-1.36 ;-0.51]$} & $7.6 \%$ \\
\hline Liu FF 2019 & 48 & -2.10 & 1.4292 & 48 & -0.90 & 1.1358 & $\div$ & -0.92 & {$[-1.34 ;-0.50]$} & $7.6 \%$ \\
\hline Lin J 2019 & 40 & -12.80 & 5.8636 & 41 & -6.82 & 6.6790 & $\frac{1}{1}$ & -0.94 & {$[-1.40 ;-0.48]$} & $6.4 \%$ \\
\hline Feng JF 2019 & 50 & -2.80 & 1.1701 & 50 & -1.50 & 1.1122 & $\longrightarrow$ & -1.13 & {$[-1.55 ;-0.71]$} & $7.5 \%$ \\
\hline An A 2019 & 40 & -2.44 & 1.0404 & 40 & -1.11 & 1.0809 & +1 & -1.24 & {$[-1.72 ;-0.76]$} & $5.9 \%$ \\
\hline \multirow{2}{*}{\multicolumn{4}{|c|}{$\begin{array}{l}\text { Fixed effect model } \mathbf{6 4 7} \\
\text { Heterogeneity: } I^{2}=50 \%, \tau^{2}=0.0530, p=0.01\end{array}$}} & 648 & & & $\diamond$ & \multirow{2}{*}{\multicolumn{3}{|c|}{$-0.98[-1.10 ;-0.87] 100.0 \%$}} \\
\hline & & & & & & & & & & \\
\hline
\end{tabular}

Figure 5 Forest plot of meta-analysis of renal function BUN difference of Bailing capsule in NS treatment. BUN, blood urea nitrogen; NS, nephrotic syndrome.

\begin{tabular}{|c|c|c|c|c|c|c|c|c|c|c|c|}
\hline \multirow{3}{*}{$\begin{array}{l}\text { Study } \\
\text { Yue YT } 2006\end{array}$} & \multirow{3}{*}{$\begin{array}{r}\text { Total } \\
28\end{array}$} & \multicolumn{3}{|c|}{ Experimental } & \multicolumn{2}{|r|}{ Control } & \multicolumn{2}{|c|}{ Standardised Mean } & \multirow[b]{2}{*}{ SMD } & \multirow{2}{*}{\multicolumn{2}{|c|}{$95 \%-\mathrm{Cl}$ Weight }} \\
\hline & & Mean & SD & Total & Mean & SD & \multicolumn{2}{|c|}{ Difference } & & & \\
\hline & & -6.46 & 13.6861 & 26 & -4.45 & 12.5811 & \multicolumn{2}{|c|}{+} & -0.15 & {$[-0.69 ; 0.38]$} & $6.6 \%$ \\
\hline Cao HJ 2009 & 34 & -8.21 & 30.1782 & 38 & -1.21 & 28.3969 & \multicolumn{2}{|c|}{ \pm} & -0.24 & {$[-0.70 ; 0.23]$} & $6.7 \%$ \\
\hline LiS 2017 & 42 & -32.00 & 16.8502 & 40 & -22.10 & 14.0865 & \multicolumn{2}{|l|}{$=$} & -0.63 & {$[-1.07 ;-0.19]$} & $6.8 \%$ \\
\hline Xiao J 2018 & 44 & -45.63 & 8.4378 & 44 & -27.86 & 8.5451 & \multicolumn{2}{|l|}{$+=$} & -2.07 & {$[-2.60 ;-1.55]$} & $6.7 \%$ \\
\hline Li WY 2018 & 41 & -48.22 & 8.6467 & 41 & -27.77 & 8.6880 & \multicolumn{2}{|l|}{ 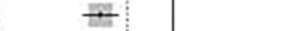 } & -2.34 & {$[-2.90 ;-1.77]$} & $6.6 \%$ \\
\hline Chen H 2018 & 50 & -45.63 & 8.4568 & 50 & -27.86 & 8.5642 & \multicolumn{2}{|l|}{+} & -2.07 & {$[-2.56 ;-1.58]$} & $6.7 \%$ \\
\hline Xiao QF 2019 & 40 & -31.70 & 25.7202 & 40 & -17.90 & 32.8818 & \multicolumn{2}{|l|}{ ㅍ } & -0.46 & {$[-0.91 ;-0.02]$} & $6.8 \%$ \\
\hline Wang XX 2019 & 45 & -64.86 & 9.3384 & 45 & -44.99 & 10.0490 & \multirow{2}{*}{\multicolumn{2}{|c|}{ \pm}} & -2.03 & {$[-2.54 ;-1.52]$} & $6.7 \%$ \\
\hline Tian HW 2019 & 47 & -55.20 & 16.2227 & 47 & -30.94 & 16.8712 & & & -1.45 & {$[-1.91 ;-1.00]$} & $6.7 \%$ \\
\hline Song JY 2019 & 50 & -36.50 & 14.9292 & 50 & -20.00 & 14.9161 & \multicolumn{2}{|l|}{$=$} & -1.10 & {$[-1.52 ;-0.68]$} & $6.8 \%$ \\
\hline Shi L 2019 & 48 & -45.76 & 8.3833 & 48 & -27.80 & 8.3700 & \multicolumn{2}{|l|}{$=$} & -2.13 & {$[-2.63 ;-1.62]$} & $6.7 \%$ \\
\hline Liu FF 2019 & 48 & -50.60 & 4.5133 & 48 & -28.90 & 4.6701 & $\mp$ & & -4.69 & {$[-5.47 ;-3.90]$} & $6.3 \%$ \\
\hline Lin J 2019 & 40 & -182.97 & 56.9178 & 41 & -100.26 & 57.7738 & \multicolumn{2}{|l|}{$t$} & -1.43 & {$[-1.92 ;-0.94]$} & $6.7 \%$ \\
\hline Feng JF 2019 & 50 & 11.48 & 7.3029 & 50 & 4.01 & 7.0669 & \multirow{2}{*}{\multicolumn{2}{|c|}{$\rightarrow$}} & 1.03 & {$[0.61 ; 1.45]$} & $6.8 \%$ \\
\hline An A 2019 & 40 & -48.94 & 7.4463 & 40 & -30.76 & 6.5307 & & & -2.57 & {$[-3.17 ;-1.97]$} & $6.6 \%$ \\
\hline \multirow{2}{*}{$\begin{array}{l}\text { Random effects model } \\
\text { Heterogeneity: } I^{2}=96 \%, \tau^{2}\end{array}$} & \multirow{2}{*}{\multicolumn{3}{|c|}{647}} & \multicolumn{3}{|l|}{648} & \multicolumn{2}{|l|}{$\infty$} & \multirow[t]{2}{*}{-1.47} & \multirow[t]{2}{*}[-2.08;-0.86]{} & $00.0 \%$ \\
\hline & & & & & & & & & & & \\
\hline
\end{tabular}

Figure 6 Forest plot of meta-analysis of renal function SCr difference of Bailing capsule in NS treatment. SCr, serum creatinine; NS, nephrotic syndrome.

treatment were reported in 15 articles. High heterogeneity existed between studies $\left(\mathrm{I}^{2}=92 \%\right)$; therefore, a randomeffects model was used to estimate the SMD. The difference in the levels of urinary protein before and 24 hours after treatment in the experimental group was significantly lower than that in the control group, with an SMD of -1.25 (95\%
CI: $-1.73,-0.78$, Figure 7).

\section{Stratified analysis}

A stratified analysis according to treatment time showed that the heterogeneity did not decrease, and no change was 


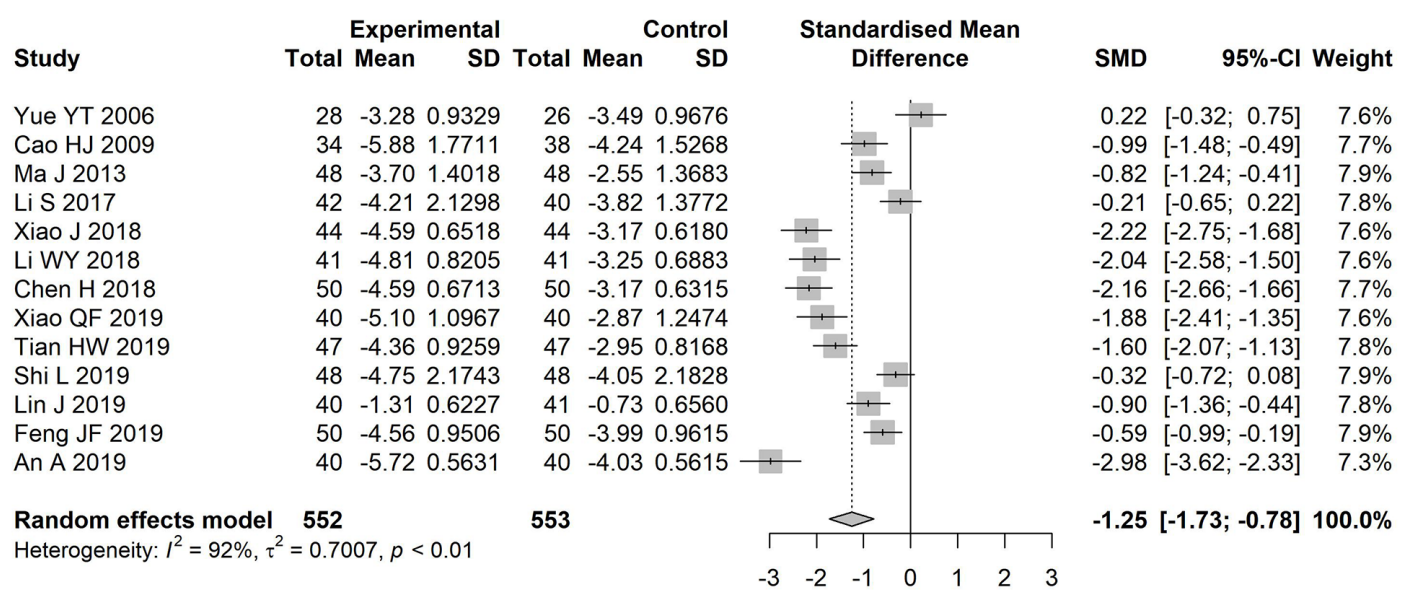

Figure 7 Forest plot of meta-analysis of renal function 24-hour urinary protein difference of Bailing capsule in NS treatment. NS, nephrotic syndrome.

Table 2 Subgroup analysis of meta-analysis of Bailing capsule treatment in NS patients

\begin{tabular}{|c|c|c|c|c|c|c|c|}
\hline Index & Subgroup & $\mathrm{n}$ & $\mathrm{I}^{2}(\%)$ & Model & $\mathrm{RR}$ & SMD & $95 \% \mathrm{Cl}$ \\
\hline Effective rate & $\geq 6 \mathrm{~m}$ & 5 & 0 & Fixed & 1.22 & - & 1.16 to 1.27 \\
\hline \multirow[t]{2}{*}{ BUN } & $<6 \mathrm{~m}$ & 11 & 62 & Random & - & -0.94 & -1.16 to -0.72 \\
\hline & $\geq 6 \mathrm{~m}$ & 3 & 0 & Fixed & - & -1.03 & -1.28 to -0.78 \\
\hline $\mathrm{SCr}$ & $\geq 6 \mathrm{~m}$ & 3 & 92 & Random & - & -1.22 & -2.13 to -0.32 \\
\hline \multirow[t]{2}{*}{ 24-hour urinary protein } & $<6 \mathrm{~m}$ & 9 & 92 & Random & - & -1.16 & -1.72 to -0.61 \\
\hline & $\geq 6 \mathrm{~m}$ & 3 & 92 & Random & - & -0.99 & -1.83 to -0.15 \\
\hline
\end{tabular}

BUN, blood urea nitrogen; SCr, serum creatinine; m, month; NS, nephrotic syndrome.

observed in the study results (Table 2).

\section{Evaluation of sensitivity and publication bias}

After eliminating the articles one by one and conducting a sensitivity analysis, the results were found to be stable, with no significant change. The results of the Egger's regression and Begg's rank correlation tests showed that publication bias existed for all indicators apart from BUN level, which had no publication bias $(\mathrm{P}>0.05)$ (Table 3).

\section{Discussion}

In NS, a large amount of protein enters the renal tubular lumen, impeding the normal function of the proximal tubule and damaging the renal tubular epithelial cells. Simultaneously, the presence of hypoproteinemia can lead to systemic edema and affect the function of the renal tubular and large amounts of proteinuria over the long term lead to renal sclerosis and fibrosis, and eventually renal failure (28). Hormones are the main drugs used to treat NS patients (29). The anti-inflammatory and immunosuppressive effects of hormones are closely interrelated. Hormones can reduce the cell number and function of T lymphocytes and monocytes/ macrophages in the circulating blood, which causes immunoglobulin synthesis to decrease and immunoglobulin decomposition to increase. At the same time, hormones can inhibit the infiltration of white blood cells and monocyte 
Table 3 Publication bias evaluation of meta-analysis of Bailing capsule treatment in NS patients

\begin{tabular}{|c|c|c|c|c|}
\hline Index & \multicolumn{2}{|c|}{ Egger } & \multicolumn{2}{|c|}{ Begg } \\
\hline Effective rate & -9.268 & 0.000 & -4.333 & 0.000 \\
\hline BUN & 0.746 & 0.469 & -0.643 & 0.520 \\
\hline $\mathrm{SCr}$ & -4.277 & 0.001 & -3.019 & 0.003 \\
\hline
\end{tabular}

BUN, blood urea nitrogen; SCr, serum creatinine; NS, nephrotic syndrome.

cells into the inflamed area and reduce the permeability of the blood vessel wall and leakage, thereby inhibiting the synthesis and release of inflammatory mediators (30).

In Chinese medicine, NS is believed to be a type of edema. It is shown in Suwen Water and Hot Points that NS originates from disorder in the kidney and the end of the lung. Suwen Zhizhenyao Dalun also points out that all edemas are related to the spleen. The pathogenesis of NS is due to three kinds of disorders including dysregulation of lung, transfer loss of the spleen, and open-and-close failure of the kidney. NS is present in the spleen and lung, and the key disorder is in the kidney. The etiology and pathogenesis of NS are complicated, and patients are susceptible to developing concurrent diseases, which worsen the NS (31). In Jingyue Quanshu Edema, every edema and other syndromes are shown to be related to the lung, spleen, and kidney. The edema belongs to extreme yin; therefore, it is the disease most closely related to the kidney. After receiving hormone treatment, the physiological balance of NS patients' bodies changes. "Qi" activity is blocked, triple energizer congestion, gasification disorder, more spleen and kidney deficiencies, and a lack of rightQi and solid guard lead to immune dysfunction and make the patients more susceptible to external disease invasion. Bailing capsules are cordyceps sinensis strains produced in China, which are mainly used to treat diseases caused by lung and kidney deficiency. Bailing capsules are reported to contain many potential biologically active ingredients, such as polysaccharides, adenosine, cordycepin, cordycepic acid, and ergosterol, which can enhance the body's immune ability, regulate hormone levels, and perform antiinflammatory and anti-hypoxia activity, thereby exerting obvious protective effects on the kidneys, lungs, liver, and other organs (32). Bailing capsules can inhibit the activation of aldose reductase, subsequently reducing the permeability of the glomerular basement membrane and reducing protein in urine (33). Furthermore, Bailing Capsules can protect $\mathrm{Na}^{+}-\mathrm{K}^{+}$-ATPase in the renal tubular and promote the production and secretion of epidermal factors from renal tubular epithelial cells. This increases the immune activity and promotes the regeneration and repair of renal tubular epithelial cells.

In this meta-analysis, NS patients in the Chinese population were included. After hormone treatment combined with Bailing capsules, it was found that the clinical efficacy in the combination treatment group was significantly better than that in control group, with a clinical efficacy 1.22 times (95\% CI: 1.16, 1.27) that of the control group. Despite the articles only containing limited data about the incidence of adverse reactions after treatment, the adverse reaction rate in the experimental group was found to be significantly lower than that in the control group, with a rate 0.22 times that in the control group (95\% CI: $0.08,0.63)$. After treatment, the renal function indicators of BUN, SCr, and 24-hour proteinuria levels were all reduced, and the degree of decrease in the renal function indexes of the experimental group after treatment was significantly larger than that of the control group, which suggests that the addition of Bailing capsules on the basis of conventional hormone therapy can significantly improve renal function and the treatment efficacy of patients, as well as reducing the incidence of adverse reactions. Analysis of subgroups according to different treatment times showed no significant changes in the results.

The results of sensitivity analysis found that the clinical effective and adverse reaction rate, and the BUN, SCr, and 24-hour urine protein levels, were relatively stable, with no single study having a significant impact on the results. Through Egger's regression and Begg's rank correlation analysis, it was found that, apart from BUN level (which had no publication bias), the reported effectiveness rates and $\mathrm{SCr}$ and 24-hour urine protein levels showed publication 
bias. When fewer than 10 articles were included, the results of the publication bias assessment study were unstable; therefore, no publication bias analysis was conducted for the adverse reactions rate. The results of this study suggest that there was a publication bias, which means that there may be some unpublished studies that have found that the conventional Western medicine combined with Bailing capsules are better than Western medicine for the treatment of NS.

The heterogeneity of the changes in the renal function indexes analysis in this study may be due to the following reasons: (I) different hospitals have different inclusion criteria for NS patients. Some studies only included primary NS (12,21,23,25-27), and some studies only showed NS without the description of the NS subtypes such as primary or secondary; (II) the characteristics of patients vary in different regions, because in some studies, NS was included with a short course of only 15 days (27), while in others, the course of NS had lasted for at least 4 years (22); (III) the Western medicine treatment plans in the control groups were also not uniform. Also, although the traditional Chinese medicine approaches all involved Bailing capsules, the dosage and treatment times varied between studies. Moreover, some studies conducted treatment for one year $(12,22)$, while in others, treatment lasted for three years (24), and in some studies, the patients were only treated for two months (18-20). The reasons above are all causes of high heterogeneity. After grouping the patients according to treatment time of $<6$ or $\geq 6$ months, we found that the changes in efficacy and renal function indexes were not related to the treatment time, and they were all significantly better than the control group. However, the heterogeneity was not significantly reduced.

This meta-analysis has the following limitations: (I) the overall quality of the included articles is poor. Except for the use of randomized controlled trials, the levels of implementation bias, follow-up bias, reporting bias, and other biases were unclear. Research bias caused by the low overall quality of the study cannot be excluded; (II) the results showed high heterogeneity. As mentioned above, the differences in characteristics of the study populations, treatment options, and treatment doses and times are all reasons for the high level of heterogeneity; (III) the sample sizes of the studies included in this analysis were generally small, especially in the studies that reported the adverse reactions after treatment. Therefore, further research is needed to confirm these findings; (IV) there was a lack of articles on which to base a comprehensive evaluation of the safety of Bailing capsules for long-term use. Therefore, further double-blind, randomized clinical trials with large cohorts comprising only patients with primary NS are needed to confirm our results.

In summary, this meta-analysis shows that conventional Western medicine combined with Bailing capsules is effective in treating NS. In our study, this treatment approach had a rate of low adverse reactions and could effectively improve the renal function of $\mathrm{NC}$ patients. However, due to the low quality and the small sample sizes of the studies included in this meta-analysis, large cohort studies and randomized double-blind clinical trials are still needed in order to comprehensively evaluate the validity and safety of Bailing capsules in NS treatment.

\section{Acknowledgments}

Funding: None.

\section{Footnote}

Reporting Checklist: The authors have completed the PRISMA reporting checklist. Available at http://dx.doi. org/10.21037/apm-20-1252

Conflicts of Interest: All authors have completed the ICMJE uniform disclosure form (available at http://dx.doi. org/10.21037/apm-20-1252). The authors have no conflicts of interest to declare.

Ethical Statement: The authors are accountable for all aspects of the work in ensuring that questions related to the accuracy or integrity of any part of the work are appropriately investigated and resolved.

Open Access Statement: This is an Open Access article distributed in accordance with the Creative Commons Attribution-NonCommercial-NoDerivs 4.0 International License (CC BY-NC-ND 4.0), which permits the noncommercial replication and distribution of the article with the strict proviso that no changes or edits are made and the original work is properly cited (including links to both the formal publication through the relevant DOI and the license). See: https://creativecommons.org/licenses/by-nc-nd/4.0/.

\section{References}

1. Heron M. Deaths: Leading Causes for 2015. Natl Vital 
Stat Rep 2017;66:1-76.

2. Zhou FD, Zhao MH, Zou WZ, et al. The changing spectrum of primary glomerular diseases within 15 years: a survey of 3331 patients in a single Chinese centre. Nephrol Dial Transplant 2009;24:870-6.

3. Han S, Yao T, Lu Y, et al. Chinese Herbal Injections for Primary Nephrotic Syndrome in Adults: A Systematic Review and Network Meta-Analysis. Evid Based Complement Alternat Med 2020;2020:1047489.

4. Tullus K, Webb HBagga A. Management of steroidresistant nephrotic syndrome in children and adolescents. Lancet Child Adolesc Health 2018;2:880-90.

5. Zhu L, Zheng ZH. Observation of clinical effect of integrated traditional Chinese and western medicine on nephrotic syndrome. Medical Forums in Basic 2016;20:4879-80.

6. Zhang JJ, Xie SY. Meta-analysis of the efficacy of Bailing capsules on primary nephrotic syndrome. The Chinese Journal of Modern Applied Pharmacy 2013;30:907-10.

7. Song HM. Observation and study of the clinical effect of Bailing capsule combined with cyclophosphamide and prednisone acetate on nephrotic syndrome. Renowned Doctor 2020;(1):246.

8. Xiao QF. Clinical observation of Bailing capsule combined with valsartan in the treatment of nephrotic syndrome. China Modern Doctor 2019;1:48-50.

9. Wang XX. Effect of Bailing capsule in adjuvant treatment of nephrotic syndrome and its influence on renal function, levels of peripheral blood 11-DH-TXB2 and HMGB-1. Clinical Research and Practice 2019;4:128-9

10. Tian HW. Effect of Bailing capsule combined with cyclophosphamide and prednisone acetate on nephrotic syndrome. J Med Theor \& Prac 2019;32:50-1.

11. Higgins JPT, Green S. Cochrane Handbook for Systematic Reviews of Interventions Version 5.1.0[EB/OL]. The Cochrane Collaboration, 2011[2012-03-30]. Available online: http:// www. Cochrane-handbook.org

12. Song JY. Clinical study on Bailing capsules combined with glucocorticoid for primary nephrotic syndrome. Journal of New Chinese Medicine 2019;0:178-80.

13. Shi L. Effect of Corbrin capsules combined with prednisone acetate on renal function and lipid metabolism disorder in patients with nephrotic syndrome. Drug Evaluation Research 2019:936-9.

14. Liu FF. Therapeutic effect of Bailing capsule, cyclophosphamide combined with prednisone acetate on nephrotic syndrome. China Health Care \& Nutrition 2019;29:153.
15. Lin J, Ye Q, Zhan LD, et al. Bailing capsules joined with mycophenolate mofetil in treating 40 cases of nephrotic syndrome. Western Journal of Traditional Chinese Medicine 2019;32:76-8.

16. Feng JF. Therapeutic effect of Bailing capsule combined with bonibizon acetate on patients with nephrotic syndrome. Journal of Liaoning University of Traditional Chinese Medicine 2019:209-12.

17. An A. Effect of Bailing capsules in adjuvant treatment of nephrotic syndrome on renal function. Medical Diet. Therapy and Health 2019:179-80.

18. Xiao J. Effect of Bailing Capsule on adjuvant treatment of nephrotic syndrome and its influence on renal function. J Med Theor \& Prac 2018;31:1168-9.

19. Li WY, Zhang Y, Jiang J, et al. Effect of Bailing tablets combined with cyclophosphamide and prednisone acetate on nephrotic syndrome. Renowned Doctor 2018;135.

20. Chen H, Chen ZZ, Huang YP, et al. Effect of Bailing Capsule on adjuvant treatment of nephrotic syndrome and its influence on renal function. Chronic Pathematology Journal 2018;19:321-2, 325.

21. Li S, Wang L. The clinical observation of corbrin capsule to treated primary nephrotic syndrome. Guide of China Medicine 2017;15:26-8.

22. Xu DY. Clinical analysis of Bailing capsule combined with benazepril in adjuvant treatment of nephrotic syndrome. Front Med 2016;6:109-10.

23. Li SM. Influence of combination of atorvastatin and Bailing capsule on renal function in patients with primary nephrotic syndrome. Anti-infection Pharmacy 2016;13:1094-6.

24. Ma J, Chen L. Clinical observation on nephrotic syndrome treated combined Beraxeprll and Balling capsule.

Chinese Medicine Modern Distance Education of China 2013;11:33-5.

25. Cao HJ. Study of low molecular weight heparin combined with Bailing capsule on treatment of primary nephrotic syndrome. China Pharmacist 2009:1100-1.

26. Yue YT, Sun ZL, Li ZB. Study on the influence of Bailing to the function of immunological and renal tubular of nephritic syndrome. Chinese Journal of Basic Medicine In Traditional Chinese Medicine 2006;12:924-5.

27. Bao JL, Lu CH, Li J, et al. Clinical observation on 68 cases of primary nephrotic syndrome treated by intracapsular injection of Bailing capsule and methylprednisolone. Chinese Journal of Integrated Traditional and Western Nephrology 2004;5:522-3.

28. Li S, Yang H, Guo P, et al. Efficacy and safety of 
immunosuppressive medications for steroid-resistant nephrotic syndrome in children: a systematic review and network meta-analysis. Oncotarget 2017;8:73050-62.

29. Wang XF, Xu YQ, Li CX, et al. Research progress of traditional Chinese medicine in the treatment of nephrotic syndrome. Guide Journal of TCM 2018;24:100-3, 116.

30. Hejazian SM, Zununi Vahed S, Moghaddas Sani H, et al. Steroid-resistant nephrotic syndrome: pharmacogenetics and epigenetic points and views. Expert Rev Clin Pharmacol 2020;13:147-56.

Cite this article as: $\mathrm{Xu} \mathrm{H,} \mathrm{Li} \mathrm{X,} \mathrm{Yuan} \mathrm{X,} \mathrm{Yuan} \mathrm{Q,} \mathrm{Chen} \mathrm{W,}$ Peng Z, Xiao X, Zhou Q. A meta-analysis of the clinical efficacy and safety of Bailing capsules in the treatment of nephrotic syndrome. Ann Palliat Med 2020;9(5):3170-3181. doi: 10.21037/apm20-1252
31. Wang G, Chen YP, Zou YQ. Modern Chinese nephrology 2003, Beijing.

32. Jiang WF. Clinical and experimental study of Bailing capsule on prevention and treatment of primary renal syndrome recurrence. Guangzhou University of TCM, 2001.

33. Liu XL, Wu YM. Research progress on the mechanism of Cordyceps sinensis in treating diabetic nephropathy. Shandong Medical Journal 2015;55:98-100.

(English Language Editor: J. Reynolds) 